\title{
Linha de cuidados em obesidade: protocolo de atenção especializada integral à obesidade e síndrome metabólica do Hospital Universitário Walter Cantídio (UFC)
}

\section{Obesity care line: a protocol describing the specialized multidisciplinary management of obesity and metabolic syndrome at the Walter Cantídio University Hospital}

Ana Flávia Torquato de Araújo Junqueira1. Virginia Oliveira Fernandes ${ }^{2}$. Joseline Maria Alves Gomes Recamonde ${ }^{3}$. Nara Fábula Costa de Brito ${ }^{4}$. Virgínia Serpa Correia Lima ${ }^{5}$. Solange Gurgel Alexandre ${ }^{6}$. Synara Cavalcante Lopes ${ }^{7}$. Renan Magalhães Montenegro Junior ${ }^{8}$.

1 Endocrinologista, mestre em Farmacologia, Serviço de Endocrinologia e Diabetes do Hospital Universitário Walter Cantídio, Universidade Federal do Ceará (UFC), Fortaleza, Ceará, Brasil. 2 Endocrinologista, doutora em Ciências Médicas, Serviço de Endocrinologia e Diabetes do Hospital Universitário Walter Cantídio, Universidade Federal do Ceará (UFC), Fortaleza, Ceará, Brasil. 3 Unidade de Nutrição Clínica, Hospital Universitário Walter Cantídio, Universidade Federal do Ceará (UFC), Fortaleza, Ceará, Brasil, Nutricionista, pós-graduanda em Transtornos Alimentares e Obesidade. 4 Psiquiatra, especialista em Terapia Cognitivo-Comportamental, Serviço de Psiquiatria do Hospital Universitário Walter Cantídio, Universidade Federal do Ceará (UFC), Fortaleza, Ceará, Brasil. 5 Psicóloga, mestre em Psicologia, Serviço de Psicologia do Hospital Universitário Walter Cantídio, Universidade Federal do Ceará (UFC), Fortaleza, Ceará, Brasil. 6 Enfermeira, doutoranda em Cuidados Clínicos em Enfermagem e Saúde, Serviço de Endocrinologia e Diabetes do Hospital Universitário Walter Cantídio, Universidade Federal do Ceará (UFC), Fortaleza, Ceará, Brasil. 7 Nutricionista, mestre em Farmacologia, Serviço de Endocrinologia e Diabetes do Hospital Universitário Walter Cantídio, Universidade Federal do Ceará (UFC), Fortaleza, Ceará, Brasil. 8 Endocrinologista, doutor em Clínica Médica, Professor Associado da Faculdade de Medicina da UFC, Serviço de Endocrinologia e Diabetes do Hospital Universitário Walter Cantídio, Universidade Federal do Ceará (UFC), Fortaleza, Ceará, Brasil.

\section{RESUMO}

A obesidade é um sério problema de saúde mundial, responsável pelo aumento na incidência de diversas outras doenças, aumento da mortalidade total, e elevação dos custos com saúde. No Brasil, onde as doenças crônicas não comunicáveis dominam o ranking de mortalidade, mais da metade da população está acima do peso. Nesse contexto, o Ministério da Saúde estabeleceu a criação das Linhas de Cuidados direcionadas às doenças crônicas de maior impacto epidemiológico. As linhas de cuidado tem como objetivo coordenar os fluxos assistenciais que devem ser garantidos ao usuário, no sentido de atender às suas necessidades de saúde de maneira integral, e reduzir a fragmentação de serviços do Sistema Único de Saúde (SUS). Este documento propõe diretrizes clínicas para a organização de uma linha de cuidados em obesidade na rede pública de Fortaleza, onde o serviço especializado e multiprofissional será oferecido no Hospital Universitário Walter Cantídio.

Palavras-chave: Obesidade. Perda de peso. Saúde pública. Protocolos clínicos. Cirurgia bariátrica. Acesso aos serviços de saúde.

\begin{abstract}
Obesity is a serious health problem worldwide, responsible for the increasing incidence of several other diseases and rising health care costs. In Brazil, where non-communicable chronic diseases dominate the mortality ranking, more than half of the population is overweight. In this regard, the Ministry of Health of Brazil established the creation of Care Lines directed to chronic diseases of major epidemiological impact. The Care Lines aim to coordinate patient flow through different complexity levels of care, to meet individuals health needs in a comprehensive manner, and to reduce fragmentation of services offered at the Brazilian public health system, the Sistema Único de Saúde (SUS). This document proposes clinical guidance for the organization of a Care Line in Obesity in Fortaleza, where the specialized and multidisciplinary services will be offered at the Walter Cantídio University Hospital.
\end{abstract}

Keywords: Obesity. Weight loss. Public health. Clinical protocols. Bariatric surgery. Health services accessibility. 
Autor correspondente: Ana Flávia Torquato de Araújo Junqueira, Rua Capitão Francisco Pedro, 1290, Rodolfo Teófilo, Fortaleza, Ceará, Brasil. CEP: 60430-370. Telefone: +55 85 3366-8165. E-mail: flaviatorq@gmail.com

Conflito de interesses: Não há qualquer conflito de interesses por parte de qualquer um dos autores.

Recebido em: 22 Dez 2015; Revisado em: 11 Jan 2016; Aceito em: 11 Jan 2016.

\section{INTRODUÇÃO}

De acordo com estimativas da Organização Mundial de Saúde (OMS), o número de adultos obesos no mundo ultrapassou meio bilhão de pessoas em 2014. ${ }^{1}$ Esse aumento alarmante na prevalência da obesidade observado mundialmente nas últimas décadas inclui o Brasil, onde $51 \%$ da população está acima do peso (IMC $25-29.9 \mathrm{~kg} / \mathrm{m}^{2}$ ) e $17,5 \%$ são obesos (IMC $\geq 30 \mathrm{~kg} /$ $\left.\mathrm{m}^{2}\right) .{ }^{2}$ Excesso de adiposidade associa-se a aumento no risco de diabetes mellitus, hipertensão, doença coronariana, câncer e mortalidade total $^{3}$ (Figura 1). O sobrepeso e a obesidade foram responsáveis por 3,4 milhões de mortes mundialmente apenas em 2010. Devido a crescente prevalência e aos reconhecidos riscos associados a obesidade, esta se tornou um sério problema de saúde global. ${ }^{4}$

Figura 1. Complicações médicas da obesidade.

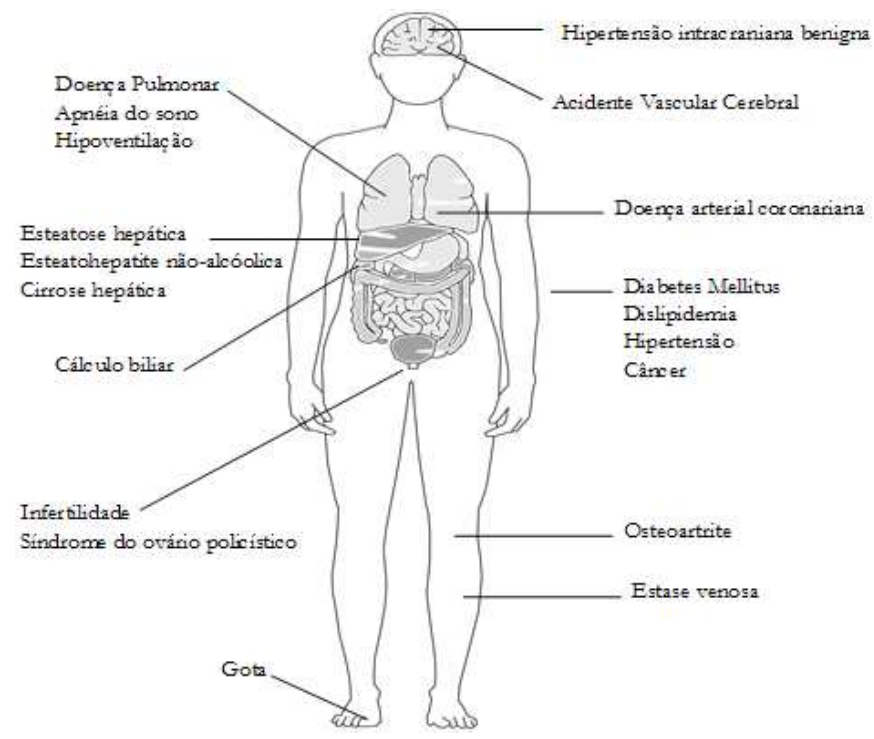

Fonte: Elaborado pelos autores.

A obesidade acarreta um aumento direto nos custos com saúde, como gastos hospitalares e com medicamentos, e também nos custos indiretos, relacionados à redução na produtividade, como ausências no trabalho, aposentadoria precoce e morte prematura da população ativa. Nos Estados Unidos, onde um terço da população é obesa, a obesidade foi responsável por um custo direto de 147 bilhões de dólares em 2008. Cada indivíduo obeso teve um custo adicional médio anual com saúde de 1.429 dólares em relação ao de peso normal. ${ }^{5}$

No Brasil, as mortes decorrentes de doenças crônicas não comunicáveis lideram as estatísticas, chegando a $72 \%$ de todas as mortes em 2010. O aumento da prevalência de obesos e, consequentemente, diabéticos e hipertensos, representa um desafio no controle da mortalidade por doenças crônicas no Brasil. Neste cenário, as políticas nacionais de saúde devem investir em metas e ações para prevenir e tratar a obesidade. ${ }^{6}$
Em 2010, a portaria n 4.279 estabeleceu as diretrizes da Rede de Atenção à Saúde, uma estratégia para superar a fragmentação e aperfeiçoar o funcionamento do Sistema Único de Saúde (SUS). Nesse sentido, a Secretaria de Atenção à Saúde propôs a construção da Rede de Atenção às Pessoas com Doenças Crônicas, com o objetivo de incentivar a mudança do modelo de atenção à saúde, garantir o cuidado integral às pessoas com doenças crônicas, prevenir e reduzir as complicações destas doenças e melhorar os indicadores de saúde.?

Em 2014, a portaria n 483 redefiniu a Rede de Atenção à Saúde de Pessoas com Doenças Crônicas e estabeleceu as diretrizes para a organização das suas Linhas de Cuidado. ${ }^{8}$ Linhas de Cuidado são definidas como uma forma de articulação de recursos e das práticas de produção de saúde, orientadas por diretrizes clínicas, entre as unidades de atenção de uma dada região de saúde, para a condução oportuna, ágil e singular, dos usuários pelas possibilidades de diagnóstico e terapia, em resposta às necessidades epidemiológicas de maior relevância. Visa à coordenação ao longo do contínuo assistencial, através da pactuação/contratualização e a conectividade de papéis e de tarefas dos diferentes pontos de atenção e profissionais. ${ }^{9}$

As linhas de cuidado expressam, portanto, os fluxos assistenciais que devem ser garantidos ao usuário, no sentido de atender às suas necessidades de saúde. A obesidade é uma das doenças crônicas priorizadas pelo Ministério da Saúde na organização das linhas de cuidado, bem como doenças renocardiovasculares, diabetes, doenças respiratórias crônicas, câncer de mama e colo de útero. ${ }^{10}$

O Hospital Universitário Walter Cantídio (HUWC) é um centro terciário que tem como missão promover o ensino, a pesquisa e a assistência especializada à saúde. A linha de cuidados em obesidade no qual participa o HUWC visa atender pacientes obesos de maior risco cardiovascular provenientes da rede de atenção básica de saúde de Fortaleza, oferecendo um tratamento integral, coordenado e multiprofissional da obesidade e suas complicações. São metas específicas do programa em relação aos usuários: promover a perda de peso e manutenção do peso perdido, promover o estilo de vida saudável, fortalecer o conhecimento do usuário sobre sua doença e ampliar sua capacidade de autocuidado, tratar e controlar as patologias associadas a obesidade, reduzir a morbi-mortalidade e melhorar qualidade de vida dos pacientes obesos. Além disso, o programa tem como objetivo promover a educação continuada de profissionais da atenção primária e profissionais em formação no hospital universitário.

A equipe multidisciplinar do HUWC conta com endocrinologistas, nutricionistas, cirurgiões bariátricos, psiquiatras, psicólogos, enfermeiros, educadores físicos e fisioterapeutas. Para seu funcionamento, a linha de cuidado 
requer uma comunicação ampla e contínua entre o serviço especializado e a atenção básica de saúde, e uma logística que facilite o fluxo de pacientes dentro dos locais de assistência. Durante a implantação desta linha de cuidados em obesidade, nossa equipe desenvolveu as diretrizes clínicas do nível especializado do cuidado e organizou um protocolo, que será resumido aqui.

\section{Critérios de triagem e encaminhamento de pacientes para o serviço especializado da linha de cuidados em obesidade}

A atenção básica de saúde tem uma tarefa essencial na estratificação, realizando o cadastramento de todos os usuários na região atendida. A estratificação de risco objetiva classificar os usuários de acordo com a gravidade da enfermidade, para definir os serviços e ações adequados em cada estrato de risco. ${ }^{10}$ Assim, devem ser encaminhados ao serviço especializado os pacientes mais complexos e com maior risco de morbi-mortalidade. A triagem e encaminhamento de pacientes atendidos pelo nível especializado da linha de cuidados em obesidade deverá, portanto, ser realizada pelas equipes da atenção básica de saúde de Fortaleza.

Deve-se usar o índice de massa corporal (IMC) para categorizar indivíduos quanto a obesidade (IMC $\geq 30 \mathrm{~kg} / \mathrm{m}^{2}$ ) e identificar adultos com risco mais elevado de mortalidade (Tabela 1). ${ }^{11}$ O IMC é calculado dividindo-se o peso (em kg) pela altura ${ }^{2}$ (metros quadrados), e, em geral, possui uma boa correlação com a quantidade de gordura corporal. ${ }^{12}$ Apesar de ser passível de falhas, pois não avalia a composição e nem a distribuição da gordura corporal, o IMC é um índice prático e fácil de ser calculado, de conhecimento difundido entre os profissionais de saúde, e que continua a ser utilizado de forma ampla mundialmente na prática clínica bem como em estudos científicos.

Deverão ser encaminhados para o HUWC indivíduos adultos ( $\geq 18$ anos) com obesidade grau 3 (IMC $\geq 40 \mathrm{~kg} / \mathrm{m}^{2}$ ), e também com obesidade grau 1 e grau 2 (IMC $\geq 30$ e $\leq 40 \mathrm{~kg}$ / $\mathrm{m}^{2}$ ) e pelo menos uma comorbidade associada à obesidade. Compreendem as comorbidades: diabetes mellitus, hipertensão arterial, dislipidemia, aumento da gordura abdominal, apnéia obstrutiva do sono, e esteato-hepatite não alcoólica (Tabela 2).

Nesta linha de cuidados, serão oferecidos tratamento clínico comportamental e cirúrgico (cirurgia bariátrica). Os critérios de encaminhamento para cirurgia bariátrica são indivíduos com obesidade grau 3 (IMC $\geq 40 \mathrm{~kg} / \mathrm{m}^{2}$ ) ou obesidade grau 2 (IMC $\geq 35$ a $39,9 \mathrm{~kg} / \mathrm{m}^{2}$ ) na presença de comorbidades relacionadas a obesidade, e histórico de falta de êxito ao tratamento clínico.

\section{Manejo clínico da obesidade}

Vários estudos clínicos demonstram melhora do controle de condições associadas a obesidade com a utilização de

Tabela 1. Classificação da obesidade em adultos de acordo com índice de massa corporal (IMC).

\begin{tabular}{lll}
\hline Classificação & IMC & Risco de comorbidades \\
\hline Abaixo do Peso & $<18.50$ & Baixo \\
Faixa normal & $18.50-24.99$ & Médio \\
Sobrepeso & $25.0-29.99$ & Aumentado \\
Obeso classe 1 & $30.0-34.99$ & Moderado \\
Obeso classe 2 & $35.0-39.99$ & Grave \\
Obeso classe 3 & $\geq 40.0$ & Muito grave \\
\hline
\end{tabular}

Fonte: Adaptado da Organização Mundial de Saúde (OMS). ${ }^{11}$

Tabela 2. Critérios utilizados na estratificação dos pacientes obesos para encaminhamento para a linha de cuidados em obesidade.

\begin{tabular}{ll}
\hline Complicação & Critérios \\
\hline Diabetes Mellitus & $\begin{array}{l}\text { Glicemia de jejum } \geq 126 \mathrm{mg} / \mathrm{dl} \text { em duas ocasiões, glicemia } \geq 200 \text { 2hs após TOTG } \\
\text { com } 75 \mathrm{~g} \text { de dextrose, glicemia aleatória } \geq 200 \text { na presença de sintomas, HA1c } \\
\geq 6,5 \% \text {, ou em tratamento para diabetes }\end{array}$ \\
$\begin{array}{l}\text { Pressão sistólica } \geq 140 \mathrm{mmHg} \text { e/ou diastólica } \geq 90 \mathrm{mmHg}, \text { ou uso de anti- } \\
\text { hipertensivo }\end{array}$ \\
$\begin{array}{l}\text { LDL } \geq 190 \mathrm{mg} / \mathrm{dl}, \mathrm{HDL} \leq 30 \mathrm{mg} / \mathrm{dl} \text {, triglicerídeo } \geq 400 \text {, ou em uso de antilipêmicos } \\
\text { Dislipidemia }\end{array}$ \\
$\begin{array}{l}\text { CA } \geq 102 \mathrm{~cm} \text { em homens e } \geq 88 \mathrm{~cm} \text { em mulheres } \\
\text { Aumento da circunferência abdominal (CA) }\end{array}$ \\
$\begin{array}{l}\text { Presença de sintomas: ronco com episódios de apnéia ou dispnéia paroxística, e } \\
\text { sonolência diurna, OU diagnosticado por polissonografia do sono }\end{array}$ \\
$\begin{array}{l}\text { Esteatose hepática ao Ultrassom e elevação de transaminases (maior risco de } \\
\text { progressão para fibrose) não explicada por hepatite viral /alcoolismo/doença } \\
\text { genética OU diagnóstico estabelecido por biópsia hepática }\end{array}$ \\
\hline
\end{tabular}

Fonte: Elaborado pelos autores. 
programas de perda de peso, incluindo diabetes mellitus tipo 2, hipertensão arterial sistêmica, dislipidemia, apnéia do sono, esteato-hepatite não alcoólica, dentre outros. ${ }^{13-17}$

A terapia da obesidade deve sempre focar em melhorar o estilo de vida e as escolhas alimentares. Mudar o estilo de vida não é fácil e os resultados nem sempre são animadores. Vários estudos mostram que muitos pacientes recuperam o peso perdido (por vezes adquirem mais) nos anos seguintes. No entanto, é possível conseguir moderada perda de peso com um programa intensivo de terapia comportamental de mudança de estilo de vida. ${ }^{18}$ Abaixo será proposto uma série de recomendações para o tratamento clínico da obesidade, baseadas em forte evidência de benefício na literatura científica, e são aplicadas no programa intensivo da linha de cuidados em obesidade.

\section{Metas de perda de peso do tratamento clínico}

- Uma meta realista e significante de perda de peso é um primeiro passo importante. Em adultos obesos com fatores de risco cardiovascular, a redução de $5 \%$ a $10 \%$ no peso inicial produz benefícios significativos como redução da hemoglobina glicada e risco de desenvolver diabetes tipo 2. ${ }^{15,17,18}$ No Diabetes Prevention Program (DPP), pacientes de alto risco engajados no programa de mudança de estilo de vida obtiveram uma perda de peso de aproximadamente $7 \%$ e uma redução de $58 \%$ na incidência de diabetes, resultado melhor que com o uso de metformina. ${ }^{19}$ Apesar de maior perda possivelmente resultar em maiores benefícios em relação a redução de fatores de risco cardiovasculares, recomenda-se uma meta de perda de 5 a $10 \%$ do peso inicial em 6 meses, por ser uma meta possível, segura, e efetiva baseada em estudos anteriores.

\section{Freqüência das visitas no tratamento clínico}

- Intervenções de alta intensidade ( $\geq 14$ visitas em 6 meses) para melhorar o estilo de vida consistindo em dieta hipocalórica, atividade física e terapia comportamental que incentive o paciente a aderir ao estilo de vida saudável, produz uma perda média de peso de $8 \mathrm{~kg}$ em 6 meses de tratamento. ${ }^{18}$ Isto aproxima uma perda média de 5 a $10 \%$ do peso inicial, que é a meta deste protocolo. Intervenção de moderada intensidade ( 1 a 2 visitas por mês) tipicamente produzem perdas médias de 2 a $4 \mathrm{~kg}$ em 6 a 12 meses. Intervenção de baixa intensidade $(<1$ visita por mês) não produzem perda de peso significativa quando comparadas com cuidado usual (intervenção mínima). ${ }^{18}$

- Recomenda-se, portanto, ao indivíduo obeso engajar-se por mais de 6 meses em um programa intensivo, com visitas frequentes, que incentive os participantes a aderir a dieta hipocalórica e atividade física. As intervenções de perda de peso de alta intensidade são realizadas individualmente, na forma de consultas, e em sessões em grupo por um profissional qualificado. O médico deve analisar junto ao paciente se este está preparado para iniciar as medidas necessárias para obter sucesso na perda de peso antes de iniciar o programa intensivo.

3. Programa intensivo de visitas da Linha de Cuidado em Obesidade (ver figura 2):

- Preconiza-se a seguinte organização de visitas no Programa intensivo, com consultas pré-agendadas, por um período de 6 meses:

\section{$\underline{\text { Semana } 1 \text { - primeiro dia: }}$}

- Consulta individual com médico endocrinologista para avaliação inicial, determinação da meta individualizada, apresentação do programa clínico, aconselhamento de dieta + atividade física, avaliação da presença de comorbidades, e

Figura 2. Fluxograma do tratamento clínico da linha de cuidados em obesidade. O tratamento intensivo por 6 meses compreende consultas individuais mensais e terapia de grupo semanais.

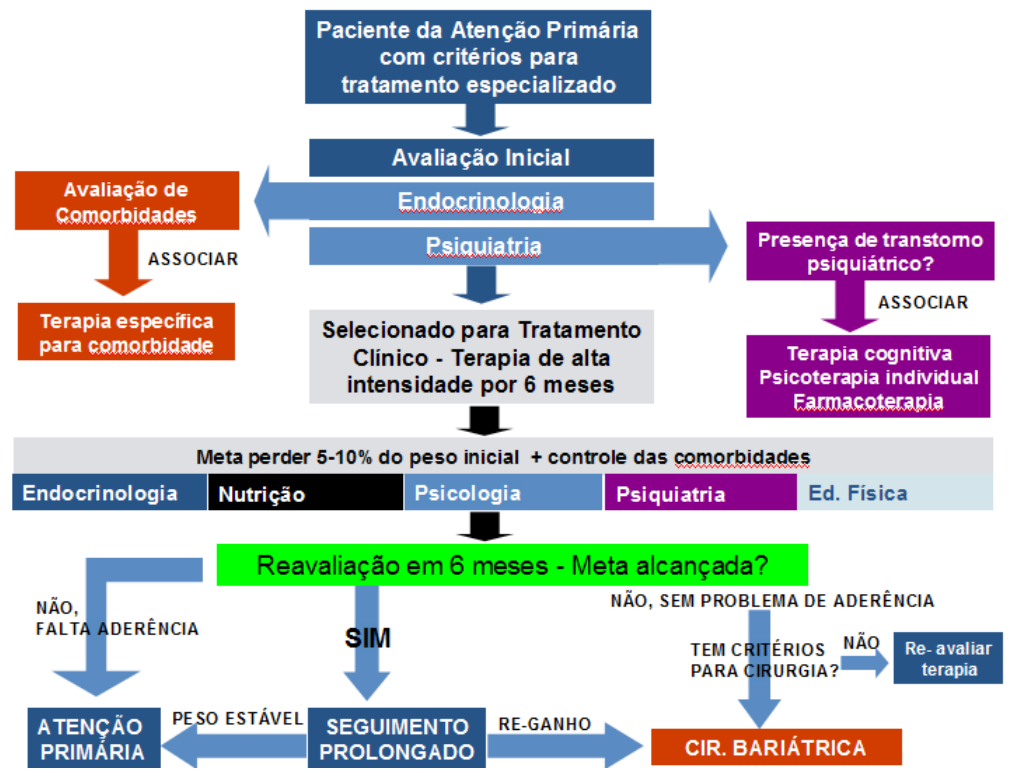

Fonte: Elaborado pelos autores. 
solicitação de exames pertinentes a cada caso.

- Consulta com médico psiquiatra para avaliação da presença de transtornos psiquiátricos, transtornos alimentares, e definição da necessidade de seguimento individual por psiquiatra/ psicólogo.

- Consulta com nutricionista para orientações alimentares e mudança do estilo de vida.

\section{Semanas 2,3,4:}

- Grupo: início da terapia comportamental em grupo, 10 a 15 pessoas, com encontros semanais, checagem e acompanhamento do peso.

\section{Semana 5, a ser repetido mensalmente:}

- Consulta com endocrinologista para mostrar exames, seguimento do peso, tratamento de condições associadas a obesidade e início de terapia medicamentosa adjuvante.

- Consulta com nutricionista para orientações alimentares e mudança do estilo de vida, acompanhamento do peso.

\section{Semanalmente, exceto uma vez por mês em dia de consulta:}

- Grupo: terapia comportamental em grupo, 10 a 15 pessoas, com encontros semanais, checagem e acompanhamento do peso.

Ao final de 6 meses, deverá ser feita a reavaliação individual das metas de perda de peso e controle de comorbidades preconizadas inicialmente.

\section{Seguimento após os seis meses iniciais:}

- Após os 6 meses iniciais, a continuidade em um programa de intervenção moderada ( 1 visita/mês) por 6 meses adicionais produz em média a manutenção da perda de $8 \mathrm{~kg}$ em ano. A continuação do tratamento no ano seguinte com visitas a cada dois meses está associada a reganho de peso de 1 a $2 \mathrm{~kg}$. Após 2 anos da intervenção de alta intensidade, 35\% a $60 \%$ dos adultos sobrepeso/obesos mantém uma perda de $\geq 5 \%$ do peso inicial. ${ }^{18}$

- Assim, após 6 meses de terapia intensiva a maioria dos pacientes irá equilibrar a ingesta calórica com gasto de energia e seu peso estabilizará. Em geral será necessário ajustes no balanço energético caso o paciente precise perder peso adicional. Intervenção contínua incluindo contato presencial após a perda de peso nos 6 meses iniciais é associada a melhor manutenção do peso perdido. Portanto, é recomendado que o indivíduo obeso que conseguiu perder peso participe de um programa de manutenção de longa duração ( $>1$ ano). $O$ programa de manutenção da perda de peso deve prover contato frequente (mensal) com profissionais treinados, para ajudar o paciente a continuar engajado em atividade física intensa (200 a 300 minutos por semana), monitorar o peso regularmente (no mínimo 1x/semana), e consumir uma dieta com reduzidas calorias necessária para manter o peso mais baixo.
Pacientes que serão seguidos no ambulatório de obesidade em programa prolongado de 1 ano:

- Pacientes que tenham perdido peso ( $\geq 5 \%$ ), mas que ainda tenham $\mathrm{IMC} \geq 30$, ou circunferência abdominal $>102 \mathrm{em}$ homens e $>88 \mathrm{~cm}$ em mulheres, ou que seja necessário melhor controle das comorbidades. Esses pacientes serão acompanhados mensalmente por 1 ano, com consultas alternadas entre médico e nutricionista, e novas estratégias para perda de peso adicional e controle das condições associadas.

- Caso o paciente tenha perdido peso ( $\geq 5 \%$ ) e o IMC esteja já abaixo de 30 após terapia intensiva de 6 meses, e as comorbidades controladas, será recomendado acompanhamento para tentar evitar reganho com consultas a cada 2 meses alternadas (médico e nutricionista) por 1 ano.

\section{Encaminhamento para cirurgia bariátrica:}

- Os critérios de encaminhamento para cirurgia bariátrica são: indivíduos com obesidade grau 3 (IMC $\geq 40 \mathrm{~kg} / \mathrm{m}^{2}$ ) ou obesidade grau 2 (IMC $\geq 35$ a $39,9 \mathrm{~kg} / \mathrm{m}^{2}$ ) na presença de comorbidades relacionadas a obesidade. ${ }^{18}$ Pacientes que preencham esses critérios podem ser encaminhados logo na primeira consulta para avaliação por cirurgião bariátrico, desde que tenham um histórico de falha ao tentar perder peso com tratamento clínico, ou que tenham tido reganho de peso após perda anterior.

- O tratamento clínico intensivo será oferecido aos pacientes que preencham critérios para tratamento cirúrgico da obesidade, mas que não desejem ser submetidos à cirurgia. Neste caso, a cirurgia pode ser recomendada novamente em um segundo momento, caso esses pacientes tenham perdido peso de forma insuficiente, ou ocorra reganho de peso após perda inicial, ou incapacidade de controlar as comorbidades.

\section{Contra-referência para rede de atenção primária de saúde:}

- Pacientes que alcançaram a meta de perda de peso de 5 a $10 \%$ e o controle das comorbidades, e mantém essa perda de peso estável por 1 ano, serão contra-referenciados para a atenção primária de saúde para continuar o acompanhamento.

- Serão contra-referenciados os pacientes que não são candidatos cirúrgicos ou que possuem contra-indicações a cirurgia bariátrica e que não conseguem perder peso $(\leq 3 \%)$ por falta de adesão a dieta/exercício/medicamento ou faltas frequentes às consultas e terapia em grupo, sem outra justificativa (ex. desordens psiquiátricas, obesidade secundária). Em geral, os indivíduos que não respondem inicialmente ao tratamento clínico intensivo da obesidade, dificilmente perderão peso com a terapia clínica nos anos subsequentes. O motivo de dar alta a estes pacientes é permitir que outros indivíduos com possibilidade de se beneficiar do tratamento especializado possam ser encaminhados a partir da atenção primária. Esses pacientes deverão continuar o manejo das comorbidades para reduzir risco cardiovascular na atenção primária; caso sinta-se motivado a perder peso, o paciente pode ser reencaminhado ao serviço especializado. 
- Pacientes que foram referenciados ao serviço especializado sem preencher os critérios de estratificação de maior risco.

7. Estrutura do programa intensivo de perda de peso utilizado na linha de Cuidados de obesidade

Os componentes principais do programa de alta intensidade para tratamento clínico da obesidade incluem: 1. Adesão a uma dieta reduzida em calorias; 2 . Incentivo à atividade física regular; 3. Uso de estratégias comportamentais para incorporação dos hábitos saudáveis.

\subsection{Educação nutricional}

- Os pacientes são acompanhados mensalmente por nutricionista para encorajar a prática de alimentação saudável, através da melhora da qualidade das escolhas alimentares e estabelecimento de comportamentos e atitudes alimentares adequados, com o objetivo de promover a perda de peso e incorporação de novos hábitos que possam ser mantidos a longo prazo.

\subsection{Atividade física}

- Atividade física: Recomenda-se atividade física de moderada intensidade (ex. caminhar rápido, dança, natação, ciclismo) por $>150$ minutos/semana; Para manutenção da perda de peso é recomendado aumento da atividade física para 200 a 300 minutos por semana. ${ }^{18}$ Os pacientes terão acompanhamento com Educador Físico para encorajar e orientar a prática de exercícios físicos. Os participantes serão também encorajados a manter-se mais ativos sempre que possível, como usar escadas ao invés do elevador.

- Indivíduos sedentários são instruídos a iniciar a atividade física aos poucos, com 10 minutos por sessão inicialmente, e progressivos incrementos até atingir a meta mínima de 150 minutos por semana.

- Os participantes com mais elevado risco cardiovascular e história de sintomatologia suspeita de isquemia miocárdica (ex. dor precordial, dispnéia aos esforços) ou doença arterial aterosclerótica (ex. claudicação intermitente) deverão ser avaliados com testes cardiovasculares antes de iniciar a atividade física de moderada intensidade.

\subsection{Intervenção cognitivo-comportamental}

- Terapia cognitivo-comportamental inclui sessões semanais em grupo com 10 a 15 participantes, com duração de 60 a 90 minutos, e com conteúdo programático pré-determinado. As sessões são conduzidas pela equipe multidisciplinar, incluindo psiquiatra, psicólogo, enfermeiro, nutricionista e educador físico. Nas sessões, são ensinados aos participantes princípios básicos de nutrição, atividade física e auto-monitoramento. As sessões focam também em desafios psicológicos, na falta de motivação, no manejo do stress, e nos problemas sociais que atrapalham a manutenção da mudança do estilo de vida em muitos pacientes.

- Os pacientes devem receber um cartão para anotação do peso e acompanhamento, bem como um diário alimentar e de atividade física. Antes de cada sessão, os pacientes são pesados individualmente e trazem o diário para checagem. A terapia cognitivo-comportamental encoraja além do automonitoramento da ingestão alimentar e atividade física, a checagem de peso regular (mínimo 1 vez por semana), e a identificação e o controle dos estímulos do ambiente que se associam a ingestão excessiva, neste sentido, pode-se aplicar a técnica de reestruturação cognitiva, que consiste em alterar convicções e pensamentos distorcidos em relação à dieta e ao peso, comumente encontrados em pacientes obesos. ${ }^{20}$

- Os pacientes que não tiverem perfil adequado para terapia em grupo e que necessitem de um acompanhamento mais individualizado (ex. na presença de distúrbio psiquiátrico) deverão ser seguidos em consultas individuais por psiquiatra e psicólogo.

\section{Tratamento medicamentoso adjuvante da obesidade}

Medicamentos podem ser usados em associação a modificação no estilo de vida como adjuvantes ao tratamento da obesidade, podendo melhorar a aderência a mudança de comportamento, e exercer um efeito aditivo na perda de peso. ${ }^{21}$ A Sociedade de Endocrinologia americana (Endocrine Society), recomenda o uso de medicação aprovada naquele país em pacientes com IMC $\geq 30$, ou $\geq 27$ associado a comorbidades. ${ }^{22}$ Neste caso, acompanhamento mensal, pelo menos inicialmente, é recomendado para avaliar eficácia e segurança dos medicamentos. Em termos de eficácia, recomenda-se manter a medicação caso seja alcançada uma perda de $5 \%$ de peso corporal em 3 meses de terapia. Caso contrário, deve-se considerar descontinuar ou substituir por uma segunda opção. ${ }^{22}$ É importante ressaltar que pode ocorrer recuperação do peso perdido caso haja descontinuação do fármaco. A ABESO (Associação Brasileira para o Estudo da Obesidade e da Síndrome Metabólica) sugere manter a medicação por longo tempo nos casos em que a resposta ao tratamento com medicação for satisfatória. ${ }^{23}$

Sobre a terapia medicamentosa nos pacientes da Linha de Cuidados em Obesidade:

- Está indicado o uso de medicações para perder peso como complemento à modificação do estilo de vida baseado na história de dificuldade de perder ou manter peso perdido com dieta e exercício apenas. A escolha da medicação deve-se basear no perfil individual do paciente considerando eficácia, custo, efeitos colaterais, condições concomitantes e presença de contra-indicações.

- É recomendável tentar limitar o uso de medicações que promovam ganho de peso como corticóides, sulfoniuréias, beta-bloquedores, anti-psicóticos (olanzapina, risperidona), anticovulsivantes (carbamazepina, divalproex, valproato), anti-depressivos tricíclicos, paroxetina, mirtazapina, lítio, etc, e dar preferência, sempre que possível, a medicações que sejam neutras em relação ao peso. Em pacientes com diabetes tipo 2, deve-se tentar usar medicações que auxiliem na perda de peso sempre que possível, como metformina, agonistas do 
GLP-1 (ex: exenatide e liraglutide), e inibidores do SGLT-2 (ex: dapagliflozina e canagliflozina), ou inibidores de DPP-4, considerados neutros em relação ao peso. ${ }^{22}$

Sobre os medicamentos disponíveis no Brasil e utilizados na Linha de Cuidados:

- Orlistate: tem ação intestinal, age inibindo lipases pancreáticas, reduzindo em $30 \%$ a absorção das gorduras ingeridas, que são eliminadas com a excreção fecal. Menos do que $1 \%$ do medicamento é absorvido e não há ação no sistema nervoso central. Acrescenta em média uma perda de $3.9 \mathrm{~kg} \mathrm{em} 1$ ano, quando comparado com placebo, e associa-se a mudanças favoráveis em relação a fatores de risco cardiovasculares. ${ }^{24}$ Tem perfil seguro para ser usado em praticamente todos os pacientes, incluindo pacientes com doença cardiovascular, sendo o alto custo o principal limitante do seu uso no nosso meio. É contra-indicado em pacientes com síndrome de má absorção crônica, colestase, ou em pacientes em uso de varfarina. ${ }^{22,23}$ Os principais efeitos colaterais são alterações gastrointestinais, como diarréia e fezes oleosas. Uso: iniciar com 120mg VO 3x/dia durante as refeições (pode-se omitir a dose do café caso seja uma refeição pobre em gordura). ${ }^{23}$

- Sibutramina: A sibutramina é um inibidor da recaptação da serotonina e da noradrenalina nas terminações nervosas do sistema nervoso central, e esta ação tem efeito anorexígeno. Quando associada a sessões de grupo para aconselhamento de mudança de estilo de vida, o uso de sibutramina levou a perda de peso média de $12,1 \mathrm{~kg}$ em um ano. ${ }^{25} \mathrm{O}$ estudo SCOUT (Sibutramine Cardiovascular Morbidity/Mortality Outcomes in Overweight or Obese Subjects at Risk of a Cardiovascular Event) incluiu 9.804 indivíduos de alto risco para evento cardiovascular, com idade média de 63,2 anos, que foram randomizados para receber placebo ou sibutramina, por um tempo médio de 3,4 anos, além dos cuidados não farmacológicos. Eventos cardiovasculares ocorreram em $11,4 \%$ dos pacientes em uso de sibutramina e em $10 \%$ dos pacientes usando placebo (um aumento de $16 \%$ no risco). ${ }^{26}$ Após a publicação desse estudo, a comercialização da sibutramina foi encerrada em vários países. No entanto, o aumento do risco de eventos cardiovasculares foi encontrado apenas em pacientes com história de doença cardiovascular e não ocorreu nos pacientes diabéticos tipo 2 sem história de doença cardiovascular. Portanto, segundo recomendações da ABESO (Associação Brasileira para o Estudo da Obesidade e da Síndrome Metabólica), a sibutramina é contra-indicada em casos de doença cardiovascular, incluindo doença arterial coronariana, acidente vascular cerebral, doença arterial periférica, hipertensão arterial não controlada, arritmia cardíaca e insuficiência cardíaca. A sibutramina não é contraindicada em diabéticos tipo 2 sem evidências clínicas de doença coronariana. ${ }^{23}$ A dose é de 10 a $15 \mathrm{mg}$ VO 1x/dia. Pode ocorrer aumento na pressão arterial sistólica e diastólica, bem como na frequência cardíaca, sendo a monitorização necessária em todas as consultas. Os efeitos colaterais mais comuns são: boca seca, constipação, cefaléia e insônia, que ocorrem em 10 a $20 \%$ dos casos. Sintomas como irritabilidade, ansiedade, náuseas e taquicardia são menos frequentes. ${ }^{23}$

Medicações utilizadas off label como emagrecedores:
- ISRS (ex. Fluoxetina e Sertralina): Inibidores seletivos da recaptação da serotonina, usados comumente para tratar depressão e distúrbios de ansiedade, não são considerados emagrecedores formais. Alguns estudos mostraram perda de peso com o uso da fluoxetina e sertralina, principalmente nos primeiros meses de uso, no entanto evidências sugerem que essas medicações não conseguem manter o peso perdido. ${ }^{27,28}$ Entretanto, podem ter papel no tratamento de pacientes com transtornos alimentares, como compulsão alimentar periódica e bulimia nervosa, especialmente quando associados a outros transtornos psiquiátricos. ${ }^{29}$ Iniciar a fluoxetina com $20 \mathrm{mg}$ VO pela manhã e, se necessário, aumentar em incrementos de 20 $\mathrm{mg}$ até a dose de $60 \mathrm{mg} /$ dia. Se for suspender, é necessário desmame lento.

- Topiramato: é um anti-convulsivante que promove perda de peso por reduzir o apetite, sendo também muito utilizado no tratamento crônico de enxaqueca. Nos Estados Unidos o topiramato de liberação prolongada foi aprovado para tratamento da obesidade em 2012, em associação com a fentermina, com uma perda de peso em torno de $10,5 \%$ após 2 anos comparada a perda de $1,8 \%$ com placebo. ${ }^{30} \mathrm{Um}$ estudo mostrou que essas medicações utilizadas em conjunto exibem um efeito aditivo, com uma perda de peso média de $9,0 \mathrm{~kg}$ para a combinação topiramato $92 \mathrm{mg} /$ fentermina $15 \mathrm{mg}$, superior a $6,4 \mathrm{~kg}$ para o topiramato $92 \mathrm{mg}$ usado isoladamente, $6,0 \mathrm{~kg}$ de perda com a fentermina $15 \mathrm{mg}$ usada isoladamente, e 1,5 $\mathrm{kg}$ de perda com o placebo. ${ }^{31} \mathrm{O}$ topiramato se mostrou benéfico em tratar casos de obesidade associados a compulsão alimentar transitória. ${ }^{32}$ Iniciar com $25 \mathrm{mg}$ a noite, pode-se aumentar até $100 \mathrm{mg} /$ dia dividido em duas tomadas (se $\mathrm{CrCl}<50 \%$ dose máx. deve ser $50 \mathrm{mg} / \mathrm{dia}$ ); Parestesias são comuns, bem como sonolência, tontura e fadiga; É teratogênico, deve ser usado com cautela em mulheres em idade fértil.

- Bupropiona: inibidor da recaptação de norepinefrina/ dopamina, a bupropiona é um anti-depressivo atípico que induz perda de peso por mecanismos não totalmente compreendidos. Tem papel também no tratamento de pacientes que desejam parar de fumar. A bupropiona de longa duração em associação com a naltrexona foi aprovada como medicação anti-obesidade nos Estados Unidos em 2014.22 Após um ano de tratamento, a combinação bupropiona 360 $\mathrm{mg}$ /dia mais naltrexona $32 \mathrm{mg} /$ dia induziu uma perda de peso significativa de 9,3\% em associação a terapia comportamental intensiva, comparada a $5,1 \%$ do grupo placebo. ${ }^{33}$ Parece haver um pequeno efeito aditivo da naltrexona em relação ao uso isolado de bupropiona. ${ }^{34}$ Pode-se iniciar bupropiona de ação prolongada $150 \mathrm{mg}$ VO 1x por dia, e aumentar a dose para $300 \mathrm{mg} / \mathrm{dia}$. Os efeitos colaterais mais comuns são insônia, náusea e boca seca.

\section{Distúrbios psiquiátricos e obesidade}

Os pacientes da linha de cuidado em obesidade serão avaliados por um médico psiquiatra. O objetivo é checar se há distúrbios psiquiátricos associados e tratar as condições existentes. $\mathrm{O}$ paciente obeso tem aumentado risco de ser diagnosticado com depressão maior, distúrbio bipolar, transtorno do pânico e agorafobia. ${ }^{35}$ Pacientes com esquizofrenia tem elevado risco 
de desenvolver obesidade devido, pelo menos em parte, ao uso de anti-psicóticos. A prevalência de síndrome metabólica nestes pacientes é de $42.6 \%$ em homens e $48.5 \%$ em mulheres, e surge em uma idade mais precoce. ${ }^{36}$ Pacientes obesos com transtorno alimentar concomitante (bulimia ou transtorno de compulsão alimentar periódica) precisam de terapia específica direcionada, e serão acompanhados por psiquiatra e psicólogo em terapia individual. Nestes casos, farmacoterapia pode também ser utilizada, como inibidores seletivos da recaptação da serotonina e topiramato, bem como sibutramina e orlistate. ${ }^{29,32}$

\section{Tratamento Cirúrgico da Obesidade}

Atualmente, a cirurgia bariátrica é o método mais efetivo e duradouro de promover perda de peso. Uma perda de $\geq 50 \%$ do excesso de peso (excesso $=$ peso total - peso ideal) é considerada sucesso cirúrgico. Em geral, a banda gástrica promove uma perda de aproximadamente $45 \%$ do excesso de peso, e o bypass gástrico de aproximadamente $60 \% .{ }^{37} \mathrm{~A}$ perda de peso é mais acentuada nos primeiros 6 meses após a cirurgia, o peso chega a um nadir em 1 a 2 anos, seguido por um período de pequeno reganho, e subsequente estabilização 8 a 10 anos após a cirurgia. ${ }^{38}$

A Cirurgia bariátrica é uma opção apropriada para adultos com IMC $\geq 40$ ou IMC $\geq 35$ com pelo menos uma comorbidade relacionada a obesidade, que não alcançam perda de peso satisfatória com manejo clínico, e no qual o risco cirúrgico não seja excessivo. ${ }^{39}$ Nos casos em que está indicada, a cirurgia bariátrica está associada com a remissão ou melhora de comorbidades associadas a obesidade e redução na incidência de diabetes, infarto do miocárdio, AVC, câncer, e mortalidade total, quando comparada ao tratamento conservador. ${ }^{40-42}$

1. Pacientes no qual a cirurgia bariátrica será indicada na linha de cuidados:

- Pacientes com IMC $\geq 40 \mathrm{~kg} / \mathrm{m}^{2}$ sem problemas médicos coexistentes ou pacientes com IMC $\geq 35 \mathrm{~kg} / \mathrm{m}^{2}$ e pelo menos uma comorbidade associada a obesidade, incluindo: diabetes tipo 2, hipertensão arterial sistêmica, dislipidemia, apnéia obstrutiva do sono, síndrome de hipoventilação, asma, esteato-hepatite não alcóolica, pseudotumor cerebral, DRGE severo, estase venosa crônica, incontinência urinária severa, osteoartrite debilitante, etc; ${ }^{39}$

- Inabilidade de perder peso ou manter o peso perdido com o tratamento conservador em tentativa anterior. Na ausência de estudos randomizados controlados que identifiquem a duração ótima do tratamento clínico antes de indicar cirurgia bariátrica, a decisão deve ser baseada em múltiplos fatores: motivação do paciente, aderência ao tratamento, risco cirúrgico e otimização das comorbidades.

2 Pacientes nos quais a cirurgia bariátrica não está indicada:

- Extremos de idade, $<16$ anos e acima de 65 a 70 anos;

- Transtornos psiquiátricos não controlados (depressão maior ou psicose não tratadas, bulimia ou desordem compulsiva alimentar periódica não tratadas;)

- Abuso de álcool ou drogas ativos;

- Condições médicas como insuficiência cardíaca severa, doença arterial coronariana instável, cirrose com hipertensão portal, doença pulmonar severa, câncer ativo, coagulopatia severa, e outras condições que acarretam elevação substancial do risco cirúrgico e anestésico;

- Inabilidade de aderência a medicações a longo prazo e limitação intelectual importante em pacientes sem suporte familiar adequado.

\section{Sobre o procedimento cirúrgico a ser escolhido:}

- Não há na literatura evidência definitiva de um procedimento em detrimento do outro, de forma que o bypass gástrico em Y de Roux (a técnica mais aplicada), o sleeve gástrico, a bandagem gástrica ajustável e a derivação biliopancreática são escolhas aceitáveis para tratar obesidade. A escolha do procedimento depende da meta a ser alcançada (perda de peso e/ou controle glicêmico), dos riscos nutricionais do procedimento, da expertise do cirurgião, da preferência do paciente, e do risco cirúrgico. A via laparoscópica é preferível em detrimento de cirurgia aberta devido a menor morbimortalidade precoce. Colescistectomia pode ser considerada concomitante ao procedimento. ${ }^{39}$

\section{Preparação pré-operatória:}

- Todos os pacientes devem ser avaliados no pré-operatório por equipe multidisciplinar, para verificar a presença de comorbidades e de possíveis causas secundárias de obesidade, estimar o risco cirúrgico, e realizar o preparo que antecede o procedimento. A avaliação deve incluir história médica detalhada, história psicossocial, exame físico e exames complementares. ${ }^{39}$

- Avaliação psicossocial: todos os pacientes devem passar por avaliação psicológica e social para avaliar presença de alterações de comportamento, doenças psiquiátricas, alcoolismo, suporte familiar, etc. Caso o paciente tenha histórico ou suspeita de transtorno psiquiátrico ativo, ou abuso de substâncias como álcool, ele deverá ser submetido a uma avaliação formal pelo psiquiatra da equipe da linha de cuidados.

- Nutricionista: Todo paciente deve passar por avaliação nutricional e se tem habilidade de incorporar mudanças nutricionais e de comportamento antes e após a cirurgia.

- A necessidade da perda de peso pré-operatória é motivo de discussão entre centros cirúrgicos mundialmente, de forma que o padrão ouro de conduta ainda não foi estabelecido. ${ }^{43}$ No nosso centro, particularmente, encorajamos a perda de peso pré-operatória de $10 \%$, por estar relacionada a mais fácil instrumentação da cavidade abdominal durante o procedimento, além de associar-se a certos resultados pós 
operatórios positivos. No entanto, a cirurgia não é contra indicada caso o paciente não consiga atingir essa meta, o que ocorre em alguns casos.

- O paciente deve ser informado sobre os riscos do procedimento, o que esperar durante o pós operatório, e a necessidade de fazer uso de medicação/reposição vitamínica pelo resto da vida após a cirurgia. A Sociedade Brasileira de Cirurgia Bariátrica e Metabólica (SBCBM) recomenda a utilização do consentimento informado antes da cirurgia bariátrica. $^{44}$

\section{- Recomendações específicas: ${ }^{39}$}

- Diabéticos: o controle glicêmico é aconselhável antes do procedimento.

- Tabagistas: cigarro deve ser evitado sempre, pacientes fumantes devem suspender o cigarro 6 semanas antes do procedimento.

- Mulheres em idade fértil: candidatas à cirurgia bariátrica devem evitar gravidez antes e por 12 a 18 meses após a cirurgia. É recomendado suspender o uso de estrogênio 1 mês antes do procedimento para reduzir o risco de eventos tromboembólicos, uma das complicações da cirurgia. Mulheres com síndrome dos ovários micropolicísticos devem ser avisadas que a fertilidade pode melhorar após o procedimento. Se for usar método contraceptivo após a cirurgia é prudente utilizar via de administração que não seja oral, caso o método utilizado na cirurgia tenha sido mal-absortivo (ex: bypass gástrico).

- Exames pré-operatórios solicitados: ${ }^{44}$

--- Laboratório: hemograma completo, coagulograma, tipagem sanguínea, ferritina, saturação da transferrina, vitamina B12, folato, 25-OHVitamina D, Cálcio total, iPTH, albumina, AST, ALT, fosfatase alcalina, GGT, bilirrubinas, eletrólitos, creatinina, glicemia de jejum, hemoglobina glicada, perfil lipídico, ácido úrico, TSH, sorologias para hepatite $\mathrm{B}, \mathrm{C}$ e HIV; Outros exames específicos a depender da avaliação do paciente.

- EDA e pesquisa de H.pilory (tratar se presente)

- Ultrassom abdominal se suspeita de esteatose hepática e litíase bilar

- Testes pulmonares: espirometria, raio $\mathrm{x}$ de tórax $\mathrm{e}$ polissonografia (se suspeita de apnéia obstrutiva do sono)

- ECG. A necessidade de testes cardíacos além de um eletrocardiograma deve ser determinada com base em fatores de riscos individuais e achados na história e exame físico. Pacientes com reconhecida doença cardiovascular precisa de consulta com cardiologista antes da cirurgia.

\section{Cuidados no período perioperatório:}

Em geral a mortalidade no período de 30 dias após a cirugia é $<1 \%$, e depende do procedimento escolhido, fatores de risco do paciente e experiência do cirurgião. As complicações precoces mais comuns incluem tromboembolismo pulmonar, fístula de anastomose, úlceras marginais, estenose, hemorragia, infecções e eventos cardíacos.

Recomendações específicas: $:^{39,45}$

- O manejo respiratório deve ser intenso e inclui espirometria, oxigênio suplementar, e instituição precoce de Pressão Positiva Contínua nas Vias Aéreas (CPAP) quando clinicamente indicado;

- É recomendado profilaxia contra trombose venosa profunda em todos os pacientes dentro das 24 hs após o procedimento. Em pacientes de alto risco, considerar ampliar o período de profilaxia. Ambular logo que possível deve ser encorajado;

- Em pacientes com diabetes, recomenda-se a monitorização glicêmica e controle com insulina, se necessário, durante a internação, para atingir uma meta glicêmica de 140 a $180 \mathrm{mg} /$ dl. Na alta hospitalar, o uso de secretagogos (sulfoniuréias e glinidas) deve ser descontinuado, se possível, e as doses de insulina ajustadas para evitar hipoglicemia no pós operatório. Metformina pode ser continuada. Em pacientes com remissão do diabetes no pós operatório, pode-se suspender as medicações e re-avaliar sua necessidade em consulta ambulatorial com endocrinologista.

- AINEs não devem ser utilizados após a cirurgia bariátrica;

- Alimentação após cirurgia bariátrica: é recomendado no pós operatório imediato de cirurgia bariátrica que os pacientes comecem a dar pequenos goles em líquidos sem açúcar dentro das primeiras $24 \mathrm{hs}$ (em geral logo que totalmente acordados). Os líquidos devem ser consumidos lentamente para evitar sintomas gastrointestinais. Os pacientes devem receber alta hospitalar apenas quando estiverem tolerando fluidos orais satisfatoriamente. É iniciado um processo gradual de progressão da consistência sobre semanas a meses, com um acompanhamento de perto da nutricionista, para permitir que o paciente se ajuste ao plano alimentar. Deve-se evitar a ingestão de alimentos e líquidos com elevada concentração de carboidratos simples para minimizar a ocorrência de sintomas gastrointestinais devido a dumping. A ingestão de proteína diária deve ser de 60 a 120 gramas, especialmente nos casos de cirurgia mal absortivas.

\section{Seguimento no pós-operatório prolongado:}

O paciente é acompanhado com visitas frequentes pelo cirurgião (para acompanhar os resultados da cirurgia e o surgimento de complicações precoces e tardias), nutricionista (para garantir adequada alimentação incluindo adequada quantidade de proteína), pelo endocrinologista (para garantir reposição vitamínica, acompanhamento das comorbidades presentes antes da cirurgia, perda e reganho de peso, complicações ósseas e metabólicas) e psicólogo (suporte psicológico, surgimento de transtornos psicológicos). O tratamento de comorbidades como DM, HAS e dislipidemia, deve seguir os consensos/guidelines em vigor (ex. meta de 
hemoglobina glicada, pressão arterial, uso de estatinas).

- Sobre o suporte nutricional suplementar:

- Recomenda-se a ingesta de uma média de 60 a 120 g de proteína diariamente para manutenção da massa magra durante a perda de peso e acompanhamento de longa duração. ${ }^{45}$ Frequentemente suplementos protéicos são necessários para atingir essa meta, o que será prescrito pelo nutricionista da equipe.

- É recomendado suplementação de vitaminas e minerais em todos os pacientes, sendo que pacientes submetidos a procedimentos mal-absortivos precisarão de maior suplementação para evitar deficiências.

- É recomendado 1 a 2 comprimidos de complexo polivitamínico com minerais por dia. ${ }^{39,45}$ Ex: Centrum ${ }^{\circledR}$, Materna ${ }^{\circledR}$ (pode-se usar vitaminas pré-natais pois tem maior quantidade de ferro e ácido fólico).

- Em geral, o polivitamínico é capaz de suprir necessidades mínimas diárias de: Vitamina A (500 mcg ou 1.600U), Vitamina E (10 mg), Vitamina K (homens 120 mcg e mulheres $90 \mathrm{mcg}$ ), Tiamina (1,2 mg/dia), Biotina (30 mcg), Folato (400 $\mu \mathrm{g} / \mathrm{dia})$, Zinco (11 $\mathrm{mg}$ em homens e $5 \mathrm{mg}$ em mulheres), Selênio (55 mcg) e Cobre (2 mg). Suplementação extra pode ser necessária e deve ser feita baseada no surgimento de deficiências. Em pacientes com vômitos frequentes, devese avaliar necessidade de suplementação extra de tiamina. Deficiência de tiamina é tratada com tiamina IV (100 a 500 $\mathrm{mg} /$ dia) por 7 dias seguida por suplementação VO $100 \mathrm{mcg} /$ dia. ${ }^{39,45,46}$

- Cálcio: A dose recomendada de cálcio elementar é de 1.200 a $2.000 \mathrm{mg}$ por dia, sendo em geral necessária a suplementação. ${ }^{39,45}$ Uma porção de leite ou derivados tem em média 200-300 mg de cálcio, com algumas fórmulas enriquecidas (ex. leite Molico ${ }^{\circledR}$ : um copo tem aproximadamente $500 \mathrm{mg}$ de cálcio). A suplementação com carbonato de cálcio mastigável, solução ou em sachês é melhor tolerado logo após a cirurgia. No entanto, citrato de cálcio é melhor absorvido na ausência de ácido do estomago.

- Vitamina D: deficiência é muito comum, embora nenhum estudo tenha sido feito com a nossa população específica, onde a exposição solar é maior e mantida o ano inteiro. A reposição deve ser feita baseada na necessidade, com meta de manter níveis séricos de $25-\mathrm{OH}$-vitamina $\mathrm{D} \geq 30 \mathrm{ng} / \mathrm{ml}$. Em geral os consensos internacionais recomendam doses altas de vitamina D. Pode-se iniciar com uma dose de $1000 \mathrm{UI} / \mathrm{dia}$, mas alguns pacientes podem precisar de doses maiores que $>50.000$ UI de colecalciferol por semana para atingir níveis adequados. ${ }^{39,45}$

- Ferro: recomendado 40 a $65 \mathrm{mg}$ de ferro elementar por dia para mulheres pré-menopausa e 15 a $30 \mathrm{mg}$ para o restante. A suplementação é feita baseada na monitorização, embora deva-se considerar iniciar suplementação empírica em mulheres com menorragia. Em alguns casos, apenas o ferro contido no complexo polivitamínico é suficiente, em outros, a reposição extra se faz necessária VO ou via parenteral, em casos refratários ou intolerância a reposição oral. Associação com vitamina C (100 a $150 \mathrm{mg}$ de ácido ascórbico) facilita absorção intestinal. ${ }^{39,45,46}$

- Vitamina B12: suplementação deve ser iniciada dentro de 6 meses após a cirurgia para evitar deficiência, e os níveis devem ser monitorados..$^{45}$ Opções de reposição são: Cianocobalamina 500 a $1.000 \mathrm{mcg}$ via oral por dia, injeção intramuscular de 1.000 mcg por mês, ou 5.000 mcg a cada 4 meses (disponível como Citoneurin $^{\circledR}$ ).

- Será realizada monitorização clínica e bioquímica periódica após a cirurgia, em média a cada 6 meses, para pesquisa de deficiências e acompanhamento dos níveis de vitaminas e minerais como ferro, vitamina B12, folato e vitamina D. Não existe indicação formal para checar sistematicamente níveis de tiamina, vitamina A, E, K, cobre, zinco e selênio em todos os pacientes, a menos que o procedimento realizado tenha sido a derivação bilio-pancreática, considerada a técnica mais malabsortiva. Assim, a deficiência destas vitaminas e minerais deve ser considerada e pesquisada na presença de evidências clínicas específicas. ${ }^{39,45}$

- Sobre o reganho de peso:

- Reganho de peso está mais comumente associado a falta de aderência a dieta e ao desenvolvimento de comportamentos alimentares mal adaptativos. É importante que os pacientes tenham acompanhamento com nutricionista e psicólogo para dar instruções sobre dieta e comportamento após a cirurgia. $\mathrm{O}$ tratamento do reganho de peso deve incluir instruções de dieta, atividade física, mudança comportamental e medicações.

- Em casos de significante e progressivo ganho de peso é recomendado avaliação se a intervenção cirúrgica do trato gastrointestinal continua anatomicamente intacta. Nestes casos, revisão da cirurgia pode ser indicada.

- Sobre o acompanhamento do metabolismo ósseo:

- Recomenda-se monitorização laboratorial (cálcio, fósforo, PTH, vitamina D, e fosfatase alcalina) a cada 6 meses, e densitometria óssea de base e 2 anos após a cirurgia. ${ }^{39}$ Bisfosfonatos podem ser considerados para tratamento de osteoporose em pacientes pós cirurgia bariátrica após adequada reposição com cálcio e vitamina D (conforme acima, em seguimento nutricional). Em geral, usa-se doses parenterais (ex: ácido zoledrônico $5 \mathrm{mg} 1 \mathrm{x} / \mathrm{ano}$ ) devido a possibilidade de reduzida absorção e potenciais complicações de erosão anastomótica. Se esta não é uma preocupação, doses semanais de alendronato $70 \mathrm{mg} / \mathrm{semana}$ ou risendronato 150 $\mathrm{mg} / \mathrm{mês}$ estão indicadas. 


\section{REFERÊNCIAS}

1. World Health Organization. Global status report on noncommunicable diseases 2014. Switzerland: WHO; 2014.

2. Malta DC, Andrade SC, Claro RM, Bernal RT, Monteiro CA. Evolução anual da prevalência de excesso de peso e obesidade em adultos nas capitais dos 26 estados brasileiros e no Distrito Federal entre 2006 e 2012. Rev Bras Epidemiol. 2014;17 Supl 1:267-276.

3. Calle EE, Thun MJ, Petrelli JM, Rodriguez C, Heath CW Jr. Bodymass index and mortality in a prospective cohort of U.S. adults. N Engl J Med.1999;341(15):1097-1105.

4. Ng M, Fleming T, Robinson M, Thomson B, Graetz N, Margono $\mathrm{C}$, et al. Global, regional, and national prevalence of overweight and obesity in children and adults during 1980-2013: a systematic analysis for the Global Burden of Disease Study 2013. Lancet. 2014;384(9945):766-81.

5. Finkelstein EA, Trogdon JG, Cohen JW, Dietz W. Annual medical spending attributable to obesity: payer-and service-specific estimates. Health Aff. 2009; 28(5):w822-31.

6. Schmidt MI, Duncan BB, Azevedo e Silva G, Menezes AM, Monteiro CA, Barreto SM, et al. Chronic non-communicable diseases in Brazil: burden and current challenges. Lancet. 2011;377(9781):1949-61.

7. Ministério da Saúde (Brasil). Documento de diretrizes para o cuidado das pessoas com doenças crônicas nas Redes de Atenção à Saúde e nas linhas de cuidado prioritárias. Brasília: Ministério da Saúde; 2012.

8. Ministério da Saúde (Brasil). Portaria $n^{0} 483$, de 1 de abril de 2014. Redefine a Rede de Atenção à Saúde das Pessoas com Doenças Crônicas no âmbito do Sistema Único de Saúde (SUS) e estabelece diretrizes para a organização das suas linhas de cuidado. Diário Oficial da União. 2014 abr 02; Seção 1(63): 50-2.

9. Ministério da Saúde (Brasil). Diretrizes para Organização das Redes de Atenção à Saúde do SUS. Brasília: Ministério da Saúde; 2012.

10. Ministério da Saúde (Brasil). Diretrizes para o cuidado das pessoas com doenças crônicas nas redes de atenção à saúde e nas linhas de cuidado prioritárias. Brasília: Ministério da Saúde; 2013.

11. World Health Organization. Obesity: preventing and managing the global epidemic: report of a WHO consultation. Geneva: WHO; 2000 .

12. Gray DS, Fujioka K. Use of relative weight and body mass index for the determination of adiposity. J Clin Epidemiol. 1991;44(6):54550

13. Schauer PR, Kashyap SR, Wolski K, Brethauer SA, Kirwan JP, Pothier CE, et al. Bariatric surgery versus intensive medical therapy in obese patients with diabetes. N Engl J Med. 2012;366:1567-76.

14. Peppard PE, Young T, Palta M, Dempsey J, Skatrud J. Longitudinal Study of Moderate Weight Change and Sleep-Disordered Breathing. JAMA. 2000;284(23):3015-21.

15. Pi-Sunyer X, Blackburn G, Brancati FL, Bray GA, Bright R,
Clark JM, et al. Reduction in weight and cardiovascular disease risk factors in individuals with type 2 diabetes: one-year results of the Look ahead trial. Diabetes Care. 2007;30(6):1374-83.

16. Huang MA, Greenson JK, Chao C, Anderson L, Peterman $\mathrm{D}$, Jacobson $\mathrm{J}$, et al. One-year intense nutritional counseling results in histological improvement in patients with non-alcoholic steatohepatitis: a pilot study. Am J Gastroenterol. 2005;100(5):107281.

17. Aucott L, Poobalan A, Smith WC, Avenell A, Jung R, Broom J, et al. Weight loss in obese diabetic and non-diabetic individuals and long-term diabetes outcomes--a systematic review. Diabetes Obes Metab. 2004;6(2):85-94.

18. Jensen MD, Ryan DH, Apovian CM, Ard JD, Comuzzie AG, Donato KA, et al. $2013 \mathrm{AHA} / \mathrm{ACC} / \mathrm{TOS}$ guideline for the management of overweight and obesity in adults: a report of the American College of Cardiology/American Heart Association Task Force on Practice Guidelines and The Obesity Society. Circulation. 2014;129(25 Suppl 2):S102-38.

19. Knowler WC, Fowler SE, Hamman RF, Christophi CA, Hoffman HJ, Brenneman AT, et al. 10-year follow-up of diabetes incidence and weight loss in the Diabetes Prevention Program Outcomes Study. Lancet. 2009;374(9702):1677-86.

20. Associação Brasileira para o Estudo da Obesidade e da Síndrome Metabólica. Diretrizes brasileiras de obesidade 2009/2010. 3. ed. Itapevi: AC Farmacêutica; 2009.

21. Wadden TA, Berkowitz RI, Womble LG, Sarwer DB, Phelan $\mathrm{S}$, Cato RK, et al. Randomized trial of lifestyle modification and pharmacotherapy for obesity. N Engl J Med. 2005;353(20):2111-20.

22. Apovian CM, Aronne LJ, Bessesen DH, McDonnell ME, Murad $\mathrm{MH}$, Pagotto U, et al. Pharmacological management of obesity: an endocrine society clinical practice guideline. J Clin Endocrinol Metab. 2015;100(2):342-62.

23. Radominski RB, Mancine $M$, Benchimol AK, Halpern A, Godoy-Mattos A, Gelonese B, et al. Atualização das diretrizes para o tratamento farmacológico da obesidade e do sobrepeso: posicionamento oficial da ABESO/SBEM - 2010. Higianápolis: ABESO; 2010.

24. Sjöström L, Rissanen A, Andersen T, Boldrin M, Golay A, Koppeschaar HP, et al. Randomised placebo-controlled trial of orlistat for weight loss and prevention of weight regain in obese patients. European Multicentre Orlistat Study Group. Lancet. 1998;352(9123):167-72.

25. Wadden TA, Berkowitz RI, Womble LG, Sarwer DB, Phelan $\mathrm{S}$, Cato RK, et al. Randomized trial of lifestyle modification and pharmacotherapy for obesity. N Engl J Med. 2005;353(20):2111-20.

26. James WP, Caterson ID, Coutinho W, Finer N, Van Gaal LF, Maggioni AP, et al. Effect of sibutramine on cardiovascular outcomes in overweight and obese subjects. N Engl J Med. 2010;363(10):90517.

27. Darga LL, Carroll-Michals L, Botsford SJ, Lucas CP. Fluoxetine's effect on weight loss in obese subjects. Am J Clin Nutr. 
1991;54(2):321-5.

28. Wadden TA, Bartlett SJ, Foster GD, Greenstein RA, Wingate BJ, Stunkard AJ, et al. Sertraline and relapse prevention training following treatment by very-low-calorie diet: a controlled clinical trial. Obes Res. 1995;3(6):549-57.

29. Reas DL, Grilo CM. Review and meta-analysis of pharmacotherapy for binge-eating disorder. Obesity (Silver Spring). 2008;16(9):2024-38.

30. Garvey WT, Ryan DH, Look M, Gadde KM, Allison DB, Peterson $\mathrm{CA}$, et al. Two-year sustained weight loss and metabolic benefits with controlled-release phentermine/ topiramate in obese and overweight adults (SEQUEL): a randomized, placebo-controlled, phase 3 extension study. Am J Clin Nutr. 2012;95(2):297-308.

31. Aronne LJ, Wadden TA, Peterson C, Winslow D, Odeh S, Gadde KM. Evaluation of phentermine and topiramate versus phentermine/ topiramate extended-release in obese adults. Obesity (Silver Spring). 2013;21(11):2163-71.

32. McElroy SL, Hudson JI, Capece JA, Beyers K, Fisher AC, Rosenthal NR, et al. Topiramate for the treatment of binge eating disorder associated with obesity: a placebo-controlled study. Biol Psychiatry. 2007;61(9):1039-48.

33. Wadden TA, Foreyt JP, Foster GD, Hill JO, Klein S, O’Neil PM, et al. Weight loss with naltrexone SR/bupropion SR combination therapy as an adjunct to behavior modification: the COR-BMOD trial. Obesity (Silver Spring). 2011;19(1):110-20.

34. Greenway FL, Dunayevich E, Tollefson G, Erickson J, Guttadauria M, Fujioka K, et al. Comparison of combined bupropion and naltrexone therapy for obesity with monotherapy and placebo. J Clin Endocrinol Metab. 2009;94(12):4898-906.

35. Simon GE, Von Korff M, Saunders K, Miglioretti DL, Crane PK, van Belle G, et al. Association between obesity and psychiatric disorders in the US adult population. Arch Gen Psychiatry. 2006;63(7):824-30.

36. Cohn T, Prud'homme D, Streiner D, Kameh H, Remington G. Characterizing coronary heart disease risk in chronic schizophrenia: high prevalence of the metabolic syndrome. Can J Psychiatry. 2004;49(11):753-60.
37. Buchwald H, Avidor Y, Braunwald E, Jensen MD, Pories W, Fahrbach K, et al. Bariatric surgery: a systematic review and metaanalysis. JAMA. 2004; 292(14):1724-37.

38. Sjostrom L, Peltonen M, Jacobson P, Sjostrom CD, Karason $\mathrm{K}$, Wedel $\mathrm{H}$, et al. Bariatric surgery and long-term cardiovascular events. JAMA. 2012;307(1):56-65.

39. Mechanick JI, Youdim A, Jones DB, Garvey WT, Hurley DL, McMahon MM, et al. Clinical practice guidelines for the perioperative nutritional, metabolic, and nonsurgical support of the bariatric surgery patient - 2013 update: cosponsored by American Association of Clinical Endocrinologists, the Obesity Society, and American Society for Metabolic \& Bariatric Surgery. Endocr Pract. 2013;19(2):337-72.

40. Sjostrom L. Review of the key results from the Swedish Obese Subjects (SOS) trial - a prospective controlled intervention study of bariatric surgery. J Intern Med. 2013;273(3):219-34.

41. Vest AR, Heneghan HM, Schauer PR, Young JB. Surgical management of obesity and the relationship to cardiovascular disease. Circulation. 2013;127(8):945-59.

42. Arterburn DE, Olsen MK, Smith VA, Livingston EH, Van Scoyoc L, Yancy WS Jr, et al. Association between bariatric surgery and long-term survival. JAMA. 2015;313(1):62-70.

43. Ochner CN, Dambkowski CL, Yeomans BL, Teixeira J, Xavier Pi-Sunyer F. Pre-bariatric surgery weight loss requirements and the effect of preoperative weight loss on postoperative outcome. Int $\mathrm{J}$ Obes (Lond). 2012;36(11):1380-7.

44. Sociedade Brasileira de Cirurgia Bariátrica e Metabólica (SBCBM). Consenso brasileiro multissocietário em cirurgia da obesidade. São Paulo: SBCBM; 2006.

45. Heber D, Greenway FL, Kaplan LM, Livingston E, Salvador J, Still C. Endocrine and nutritional management of the post-bariatric surgery patient: an Endocrine Society Clinical Practice Guideline. J Clin Endocrinol Metab. 2010;95(11):4823-43.

46. Kushner RF, Cummings S, Herron DM. Bariatric surgery: postoperative nutritional management [Internet]. Uptodate; 2014 Aug 18[acesso 2015 abr 9]. Disponível em: http://www.uptodate.com/ contents/bariatric-surgery-postoperative-nutritional-management

\section{Como citar:}

Junqueira AF, Fernandes VO, Recamonde JM, Brito NF, Lima VS, Alexandre SG, et al. Linha de cuidados em obesidade: protocolo de atenção especializada integral à obesidade e síndrome metabólica do Hospital Universitário Walter Cantídio (UFC). Rev Med UFC. 2015 juldez;55(2):63-74. 